\title{
Bone marrow examination of HIV-infected children in HAART era reveals a spectrum of abnormalities: a study from single tertiary care center of North India
}

\author{
Saniya Sharma ${ }^{1} \cdot$ Ravinder Kaur Sachdeva ${ }^{2}$ Man Updesh Singh Sachdeva ${ }^{1} \cdot$ Sreejesh Sreedharanunni ${ }^{1}$. \\ Shano Naseem ${ }^{1} \cdot$ Prashant Sharma $^{1} \cdot$ Narender Kumar $^{1} \cdot$ Jasmina Ahluwalia ${ }^{1} \cdot$ Reena Das $^{1} \cdot$ Neelam Varma ${ }^{1}$. \\ Amanjit Bal $^{3}$. Deepti Suri ${ }^{2} \cdot$ Surjit Singh ${ }^{2}$
}

Received: 28 June 2021 / Accepted: 26 September 2021 / Published online: 5 October 2021

(c) The Author(s), under exclusive licence to Springer-Verlag GmbH Germany, part of Springer Nature 2021

\begin{abstract}
Pediatric HIV is a significant contributor to childhood morbidity and mortality. As HIV infects bone marrow CD34-positive progenitor cells, the hematological abnormalities are well-expected. The viral load is much higher in children predisposing them to opportunistic infections and hematopoietic malignancies. The present study aimed to determine the spectrum of hematological abnormalities that may yield clinically useful information in HIV-infected children. This was a retrospective study of $19 \mathrm{HIV}$-infected children who underwent bone marrow examination. Overall, 20 peripheral blood and bone marrow samples were analyzed for morphological changes in all hematopoietic lineages. Immunohistochemistry was performed on trephine biopsy sections for evaluation of $\mathrm{B}$ and $\mathrm{T}$ cells and cytomegalovirus inclusions. Anemia was the most common cytopenia (95\%), followed by thrombocytopenia (45\%) and leukopenia (40\%). Myelodysplasia was noted in $70 \%$ of cases. Dysmegakaryopoiesis was predominant in $65 \%$ of cases, while dyserythropoiesis was noted in $25 \%$ of cases. Hemophagocytosis and reactive cellular changes were noted in $20 \%$ of cases each. Benign lymphoid aggregates and granulomatous inflammation were observed in $15 \%$ and $10 \%$ of cases, respectively. Significant myelofibrosis was found in $35 \%$ of cases. One case showed infiltration by Burkitt lymphoma. Parvoviral inclusions were identified in 1 case. An interstitial increase and aggregates of CD8 $+\mathrm{T}$ cells and reduced $\mathrm{CD} 4+\mathrm{T}$ cells were noted. CD20 + B cells were normal to mildly increased in the bone marrow. Bone marrow examination provides clinically significant information in pediatric HIV revealing the underlying cause of hematopoietic abnormalities and allows the exclusion of major hematological neoplasms.
\end{abstract}

Keywords Acquired immunodeficiency syndrome $\cdot$ Bone marrow $\cdot$ Human immunodeficiency virus $\cdot$ HIV myelopathy $\cdot$ Pediatric HIV

\section{Introduction}

Human immunodeficiency virus (HIV)/acquired immunodeficiency syndrome (AIDS) in the pediatric population contributes significantly to childhood morbidity and mortality. Out of an estimated total number of 2.14 million people living with HIV (PLHA) in India in 2017, children below 15 years of age constituted 61,000 [1]. Vertical transmission is the most common mode of spread in more than $90 \%$ of cases in our country owing to the higher number of normal

Man Updesh Singh Sachdeva

drmanupdeshpgi@yahoo.co.in

Extended author information available on the last page of the article vaginal deliveries and breastfeeding [2]. HIV primarily infects CD4 + T cells in peripheral blood and lymphoid tissues. Hematopoietic CD34-positive progenitor cells in bone marrow may also be infected, manifesting as hematological abnormalities, chiefly cytopenia and myelodysplasia [3]. Similar to adults, peripheral blood viral load correlates with the degree of CD4 + T cell lymphopenia and CD4/CD8 ratio in children, and profound reduction in immunity predisposes them to opportunistic infections and malignancies [4]. Anemia is the most common cytopenia, though neutropenia and thrombocytopenia are also frequent [4]. Peripheral blood and bone marrow viral load are much higher, and the disease follows a much more aggressive course in HIV-infected children as compared to adults owing to the severe loss of cell-mediated and humoral immunity [4]. Bone marrow 
examination is indicated to investigate the cause of cytopenia, to analyze cellular and architectural changes associated with HIV infection and coexistent opportunistic infections, and for the staging of HIV-associated lymphoma. The spectrum of diseases affecting bone marrow has also changed with the better availability of antiretroviral therapy (ART). In addition, regional factors existing in developing countries like India may also contribute to diseases affecting bone marrow. We evaluated morphological changes in bone marrow with an intent to provide insight into the spectrum of pathological changes in HIV-infected children from North India.

\section{Material and methods}

Retrospective screening of the files of 500 HIV-seropositive children revealed 23 children who underwent bone marrow examination between January 2014 and December 2018. The clinical and hematological data were available for 19 children. Demographic and clinical information available from the clinical database was recorded that included presenting complaints, indications of bone marrow examination, CD4 + T cell counts, ART status, and opportunistic infections. The clinical indication of bone marrow examination together with peripheral blood and bone marrow abnormalities was analyzed in each case. In one case, bone marrow was done twice; therefore, 20 peripheral blood and 20 bone marrow samples were analyzed. Two May Grunwald Giemsa (MGG) stained aspirate smears, 2 trephine imprint smears, and 1 hematoxylin and eosin (H\&E) stained biopsy section were analyzed in each case. Reticulin staining was performed in 9 cases for assessment of myelofibrosis based on European Myelofibrosis Network (EUMNET) grading system. Ziehl-Neelsen (ZN) staining was performed for detection of acid-fast bacilli (AFB) in 4 cases with a clinical diagnosis of tuberculosis and atypical mycobacterial infection. Since 4 cases had a clinically evident fungal infection, periodic acid-Schiff (PAS) staining was performed for identifying fungal elements. Immunohistochemistry (IHC) for CD20+B cells, CD4 + T cells, and CD8 + T cells and cytomegalovirus (CMV) was performed on trephine biopsy sections in 14 cases. Hematology cell counter Coulter LH780 (Beckman Coulter, Miami, FL, USA) was used to perform a complete blood count (CBC). CD4 + T cell counts were performed on BD FACSCalibur flow cytometer (BD, Biosciences, USA). IHC was performed on automated immunostainer Ventana BenchMark XT. Antibodies used were as follows: anti-CD4 rabbit monoclonal primary antibody, clone SP35, Ventana; anti-CD20 primary antibody, clone L26, Ventana; anti-CD8 monoclonal mouse anti-human antibody, clone C8/1448, Dako, 1:50; and monoclonal mouse anti-cytomegalovirus, clone CCH2 + DDG9, Dako, 1:50.
Informed consent was taken before performing the bone marrow procedure. The study was approved by the Institute Ethics Committee (INT/IEC/2021/SPL-287).

\section{Results}

Nineteen HIV-infected children with ages ranging from 3 months to 14 years, including 1 child with 2 consecutive bone marrow procedures, were finally included in the study. The male to female ratio was 3.75:1. Absolute CD4+ T cell count ranged from 2 to $864 / \mu$ l. Of 19 children, 14 were being treated with ART, and 5 were treatment-naive. Opportunistic infections were diagnosed in 13 children (68.4\%) [isolated CMV $(n=2)$, Pseudomonas and CMV $(n=1)$, Pneumocystis jirovecii and Enterococcus $(n=1)$, Candida and CMV $(n=1)$, Mycobacterium tuberculosis and CMV $(n=1)$, Mycobacterium tuberculosis alone $(n=2)$, atypical mycobacterial infection $(n=1)$, aspergillosis $(n=1)$, candidiasis $(n=2)$, Enterococcus with Molluscum contagiosum $(n=1)]$. Clinically, the majority of patients had fever $(63.2 \%)$ followed by hepatosplenomegaly (47.4\%), lymphadenopathy (31.6\%), progressive pallor (21\%), cough and rapid breathing $(21 \%)$, and gastrointestinal symptoms like abdominal pain and distention, vomiting, diarrhea, dysphagia, and odynophagia (21\%). Two patients had presented with neck and abdominal swelling, respectively, while central nervous system manifestations (seizures and altered sensorium) and epistaxis were present in 1 case each. Clinical indications of bone marrow examination were cytopenia (57.9\%), opportunistic infections (52.6\%), hemophagocytic lymphohistiocytosis (31.6\%), staging of lymphoma (21\%), and drug-induced myelosuppression (5.2\%).

Overall, cytopenias were found in $100 \%$ cases comprising of pancytopenia in $20 \%$ cases, bi-cytopenia, and single lineage cytopenia in $40 \%$ each. Of all cytopenias, anemia (hemoglobin: 3.6 to $10.6 \mathrm{~g} / \mathrm{dl}$ ) was most common accounting for $95 \%$ of all cases followed by thrombocytopenia (platelet count: 3.0 to $143 \times 10^{3} /$ microliter) in $45 \%$ and leukopenia ( 0.9 to $4.4 \times 10^{3} /$ microliter) in $40 \%$ cases with 2 cases each having both neutropenia and thrombocytopenia. Microcytic hypochromic anemia was predominant (57.9\%), followed by normocytic normochromic anemia (26.3\%) and macrocytic anemia (15.8\%). Lymphopenia was present in 35\%, whereas neutropenia was present in $20 \%$ of cases. In one case, each had leukocytosis and thrombocytosis. Hypersegmented neutrophils (HSPs) with $\geq 6$ nuclear lobes and/or 5 lobes in at least $3 \%$ cells were found in $30 \%$ of cases. Large granular lymphocytes (LGLs) were found to be increased in $25 \%$ cases. The leukoerythroblastic reaction was noted in 2 cases (10\%), one of which showed marrow infiltration by Burkitt lymphoma and the other had sepsis with pneumonia. Additionally, atypical lymphoid cells with deeply basophilic 
and vacuolated cytoplasm were noted in 1 case who was diagnosed with Burkitt lymphoma (Table 1).

Bone marrow cellularity varied from hypocellular (35\%) to normocellular (35\%) and hypercellular (30\%). The increase in the number of megakaryocytes was observed in $30 \%$ of cases, and $10 \%$ of cases showed a decrease. Myelodysplasia was found in $70 \%$ of cases. Dysmegakaryopoiesis in the form of small hypolobated and monolobated forms exhibiting nuclear hyperchromasia and swollen nuclei with several naked nuclei predominant in $65 \%$ of cases (Fig. 1a), while dyserythropoiesis (nuclear budding, irregularity, and multinucleation) was found in 25\% cases. Megaloblastic erythropoiesis was reported in $45 \%$ of cases. Erythroid hypoplasia with an increase in myeloid to erythroid ratio was found in $20 \%$ of cases. Giant myelocytes and metamyelocytes with hypergranular cytoplasm were present in $30 \%$ of cases, though no significant dysplasia was noted in granulocytic lineage. Hemophagocytosis (Fig. 1b) and reactive cellular changes (histiocytic proliferation and plasmacytosis) were noted in 4 (20\%) cases each. Benign lymphoid aggregates and granulomatous inflammation (Fig. 1c) were observed in $3(15 \%)$ and $2(10 \%)$ cases, respectively, while significant myelofibrosis ( $\geq$ grade 2 ) on reticulin staining was found in 7 (35\%) cases, and mild fibrosis (grade 1) was noted in 2 cases (10\%) (Fig. 1d). ZN staining performed in 4 cases showed AFB positivity in 1 case only $(25 \%)$. PAS staining performed in 4 cases did not reveal any fungal profiles. Only 1 case (5\%) showed diffuse infiltration by medium- to large-sized atypical lymphoid cells with prominent nucleoli, opened up chromatin, and scanty to moderate vacuolated cytoplasm along with numerous macrophages, consistent with infiltration by Burkitt lymphoma
(Fig. 1e). Morphological features of parvovirus infection in the form of giant proerythroblasts, intranuclear inclusions, and cytoplasmic blebs along with erythroblastopenia (Fig. 1f) were identified in 1 case (5\%); however, none of the cases revealed CMV inclusions or cytopathic changes indicative of CMV infection (Table 2). Perls' stain revealed low iron stores (grade 0 ) in 3 (25\%) cases out of 12 marrows with evaluable particles. On immunohistochemical analysis (Table 3), interstitial increase and aggregates of CD8 $+\mathrm{T}$ cells in $57.1 \%$ of cases and an overall reduction in $\mathrm{CD} 4+\mathrm{T}$ cells in $78.4 \%$ of cases were noted. CD20 $+B$ cells showed a normal distribution to mild interstitial increase [Fig. 2]. Immunostaining for CMV did not reveal any inclusions. Clinical and hematological data of 19 HIV-infected children is depicted in supplementary Table 1.

\section{Discussion}

Hematological manifestations in HIV-infected children may be due to HIV infection per se, drug-induced myelosuppression, opportunistic infections, and HIV-related neoplasms and usually correlate with disease severity and clinical stage. Of all abnormalities, anemia was the most common (95\%) in our study, the reported frequencies being $66 \%, 69 \%, 77 \%$, and $92 \%$ in previous studies [5-8]. However, contrary to previous studies, microcytic hypochromic anemia (57.9\%) was the predominant subtype. This could be attributed to anemia of inflammation or chronic disease in later stages of HIV or due to nutritional iron deficiency in our setting, though only 3 of 12 cases showed low iron stores on Perls' stain. Anemia of chronic disease could explain normocytic
Table 1 Peripheral blood cell abnormalities $(n=20)$

\begin{tabular}{llll}
\hline Peripheral blood cell abnormalities & & $n(\%)$ & \\
\hline Cytopenia & Single lineage & $20(100)$ & $8(40)$ \\
& Bi-cytopenia & $8(40)$ \\
& Pancytopenia & $4(20)$ \\
Anemia & Microcytic hypochromic & $19(95)$ & $11(57.9)$ \\
& Normocytic normochromic & & $5(26.3)$ \\
& Macrocytic & & $3(15.8)$ \\
Leukopenia* & & $8(40)$ & \\
Neutropenia & & $4(20)$ & \\
Lymphopenia & & $7(35)$ & \\
Thrombocytopenia & & $9(45)$ & \\
Leukocytosis (neutrophilia) & & $1(5)$ & \\
Thrombocytosis & & $1(5)$ \\
Hypersegmented neutrophils & $6(30)$ \\
Increase in large granular lymphocytes & & $5(25)$ \\
Leukoerythroblastic reaction & $2(10)$ \\
Atypical lymphoid cells & $1(5)$ \\
\hline
\end{tabular}

*Two cases had both neutropenia and lymphopenia 


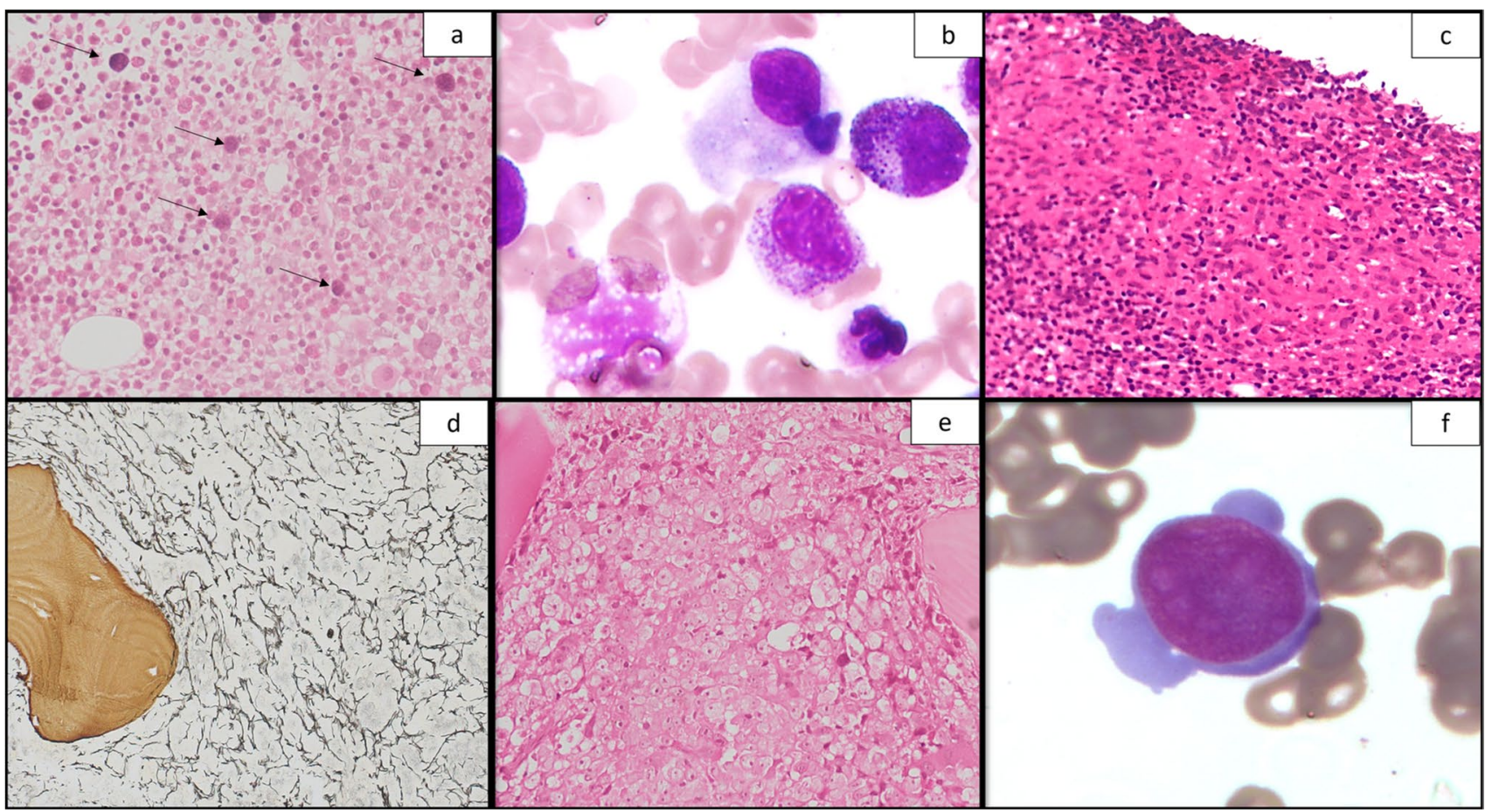

Fig. 1 Panel of microphotographs showing bone marrow findings. a Cellular bone marrow spaces with prominent dysmegakaryopoiesis, showing small hypolobated megakaryocytes and naked hyperchromatic megakaryocytic nuclei (arrows) (hematoxylin \& eosin stain, original magnification $400 \times$ ); b hemophagocytosis in a CMV positive case (May Grunwald Giemsa stain, original magnification $1000 \times)$; $\mathbf{c}$ granulomatous inflammation in a case of pulmonary tuber- culosis (hematoxylin \& eosin stain, original magnification $400 \times$ ); d reticulin fibrosis (reticulin stain, original magnification $100 \times$ ); e infiltration by Burkitt lymphoma (hematoxylin \& eosin stain, original magnification $200 \times$ ); $\mathbf{f}$ giant proerythroblast with intranuclear inclusion and cytoplasmic blebs, consistent with parvovirus infection (May Grunwald Giemsa stain, original magnification $1000 \times$ )
Table 2 Bone marrow abnormalities $(n=20)$

\begin{tabular}{llll}
\hline Morphological findings & & $n(\%)$ & \\
\hline Cellularity & Normocellular & $7(35)$ \\
& Hypercellular & $6(30)$ \\
Myelodysplasia & Hypocellular & $7(35)$ \\
& Dysmegakaryopoiesis & $14(70)$ & $13(65)$ \\
Granulocytic lineage & Dyserythropoiesis & $5(25)$ \\
Hemophagocytosis & hypergranulation & \\
Myelofibrosis & & $6(30)$ & \\
Granulomatous inflammation & & $4(20)$ & \\
Lymphoma infiltration (Burkitt lymphoma) & & $7(35)$ & \\
Viral inclusions (parvovirus) & & $2(10)$ & \\
Benign/reactive lymphoid aggregates & & $1(5)$ & \\
Reactive changes (increased histiocytes and plasmacytosis) & $1(5)$ & \\
\hline
\end{tabular}

normochromic anemia (26.3\%), whereas macrocytic anemia $(15.8 \%)$ was possibly due to antiretroviral therapy (ART) as these children were receiving zidovudine-based ART [6]. Of these 3 patients, vitamin B12 and folate levels were tested in 1 patient and were normal. Other important causes of anemia in HIV include direct HIV infection of erythroid progenitor cells, reduced erythropoietin levels, Parvovirus B19, tuberculosis and atypical mycobacterial infections, cytokine production inhibiting the hematopoiesis, and antierythrocyte antibodies [6]. Thrombocytopenia was present 
Table 3 Immunohistochemical assessment of CD4+and CD8+T cells and CD20 $+\mathrm{B}$ cells $(n=14)$

\begin{tabular}{llll}
\hline Lymphocytes & $\begin{array}{l}\text { Normal/intersti- } \\
\text { tial distribution }\end{array}$ & Decreased & Increased/aggregates \\
\hline $\mathrm{CD} 4+\mathrm{T}$ cells & $2(14.3 \%)$ & $11(78.6 \%)$ & $1(7.14 \%)$ \\
$\mathrm{CD} 8+\mathrm{T}$ cells & $4(28.6 \%)$ & $2(14.3 \%)$ & $8(57.14 \%)$ \\
$\mathrm{CD} 20+\mathrm{B}$ cells & $9(64.3 \%)$ & 0 & $5(35.7 \%)$ \\
\hline
\end{tabular}

in $45 \%$ of cases in our study which is quite high as compared to the reported frequency in the literature [6]. It could be due to drug-induced myelosuppression, immune-mediated and direct HIV infection of megakaryocytes [6]. Lymphopenia $(35 \%$, in our study) due to cell death of CD4 + T cells and in late stages of CD8 $+\mathrm{T}$ cells is the most characteristic abnormality of HIV/AIDS. Absolute lymphocyte count may be used as a surrogate marker of immune status [6]. Neutropenia ( $20 \%$ in our study) usually results from impaired hematopoiesis or immune-related mechanisms [6]. Other important peripheral blood findings included increased LGLs in 25\% and HSPs in 30\% cases. LGL proliferation has been reported in post-viral infections (CMV and HIV) as a response to chronic antigen stimulation of cytotoxic T-cells and is usually polyclonal [9]. Of 5 cases with LGL expansion, CMV infection was present in 2 cases, and $\mathrm{Mol}$ luscum contagiosum and oral thrush were present in 1 case each, in addition to underlying HIV infection. Of 6 cases with HSPs, 5 were receiving ART, and 4 showed megaloblastic changes in erythroid precursors along with giant myelocytes and metamyelocytes. These changes could be explained based on megaloblastic hematopoiesis and impaired DNA and RNA synthesis due to ART intake [10]. Vitamin B12 and folate levels were not available in these patients. In a recent study on 255 HIV-infected children, peripheral cytopenia was present in $38.8 \%$ of cases, most common being anemia $(21.2 \%)$ followed by leukopenia (12.2\%), lymphopenia (11\%), bi-cytopenia (anemia and leukopenia: $3.9 \%$ ), and thrombocytopenia (1.6\%), respectively. The factors that were strongly associated with anemia were age between 2 and 10 years, high viral load, zidovudinebased ART, and insufficient intake of green vegetables by these children [11].

Bone marrow aspirate and biopsy revealed a heterogeneous but clinically useful morphological diagnosis in HIV. In contrast to previous studies, bone marrow in the present study was predominantly hypocellular $(35 \%)$ and normocellular (35\%) followed by hypercellular (30\%). Out of 7 cases with hypocellular marrow, 4 had bi-cytopenia, 2 had anemia, and 1 had pancytopenia. Hypocellular marrow could

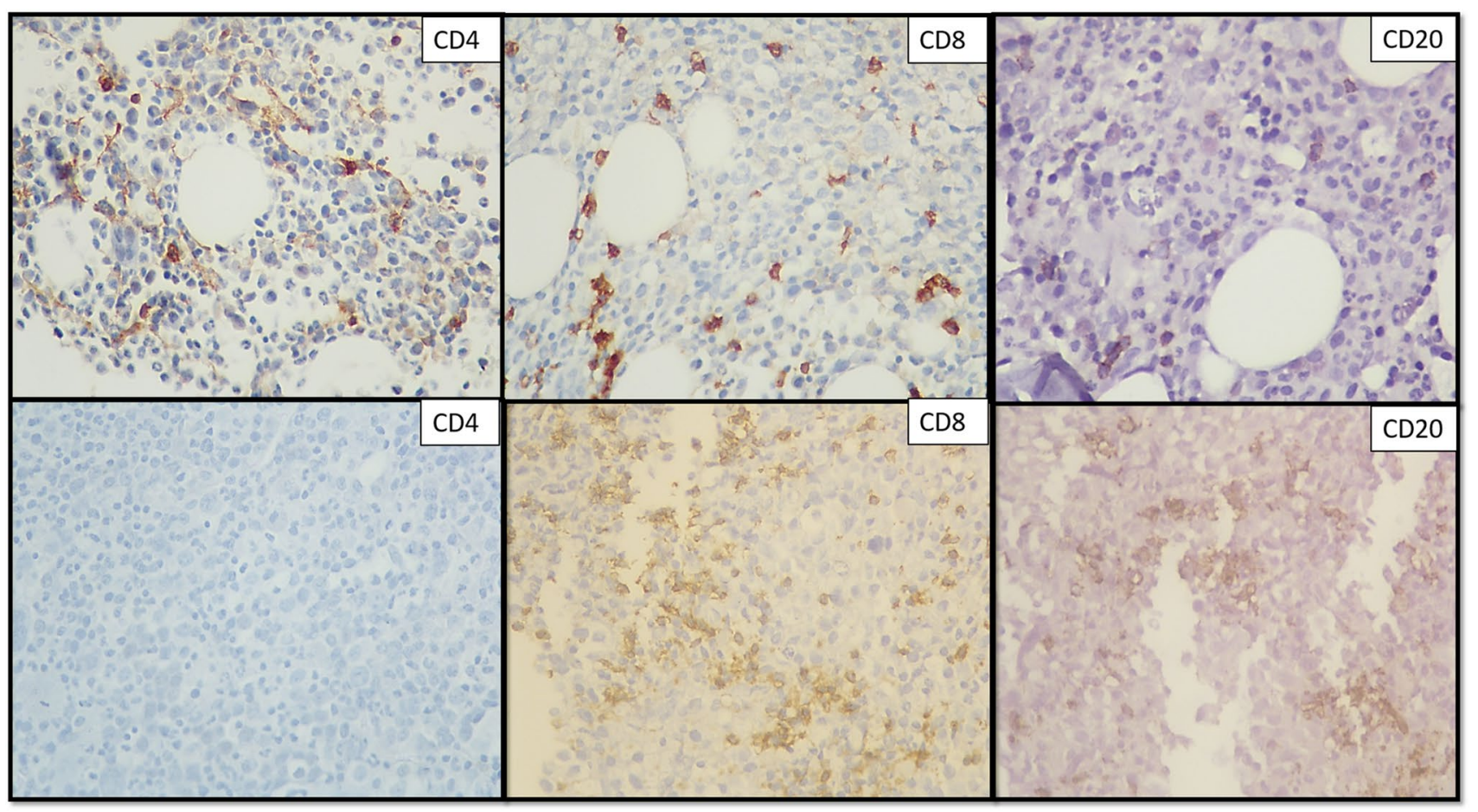

Fig. 2 Panel of microphotographs from bone marrow trephine biopsy sections showing interstitial distribution of $\mathrm{CD} 4+\mathrm{T}$ cells, CD8 $+\mathrm{T}$ cells, and CD20 + B cells in a HIV-seronegative pediatric Hodgkin lymphoma for staging (upper panel), compared with HIV-seropos- itive case (lower panel) showing reduced $\mathrm{CD} 4+\mathrm{T}$ cells, increased $\mathrm{CD} 8+\mathrm{T}$ cells (aggregates), and increased CD20 + B cells (immunohistochemical stain, original magnification $200 \times$ ) 
be due to advanced disease stage with direct HIV infection of CD34-positive progenitor cells, drug-induced and opportunistic infections leading to profound myelosuppression [12]. In cases with hypocellular marrow, a history of drug intake was present in 3 cases, and 6 cases had opportunistic infections (CMV and Pseudomonas:1, CMV and Candida:1, Pneumocystis jirovecii and Enterococcus: 1, atypical mycobacterial infection:1, CMV:1, parvovirus:1). In cases with hypercellular marrow, morphological changes included increased megakaryocytes with dysmegakaryopoiesis in 3 cases, trilineage hyperplasia in 1 case, erythroid hyperplasia with megaloblastic change in 2 cases, myelofibrosis in 4 cases, multiple epithelioid granulomas in 1 case, increase in histiocytes in 2 cases, and infiltration by Burkitt lymphoma in 1 case. Hypercellular marrow may be attributed to the initial disease stage associated with increased megakaryopoiesis, dyshemopoiesis, plasma cell hyperplasia, and reactive lymphoid infiltrates, highly suggestive of HIV infection [12-14]. In our study, another interesting finding was myelodysplasia (70\%) including dysmegakaryopoiesis (65\%) and dyserythropoiesis (25\%); however, no significant dysgranulopoiesis was noted except for giant myelocytes and metamyelocytes (30\%). Hematopoiesis associated with HIV infection is usually dysplastic with abnormal maturation. These changes are non-specific and do not correspond to the well-established criteria of myelodysplastic syndrome (MDS) and are termed as HIV myelopathy [14]. Myelodysplastic changes in all 3 hematopoietic lineages have been described in several studies in pediatric HIV/AIDS. Dysplasia in granulocytes and megakaryocytes may be prominent even in the absence of drug therapy [14]. Dysgranulopoiesis in the form of giant metamyelocytes with irregular nuclei and opened up chromatin, HSPs, and hypogranular cytoplasm has been reported [14]. Dysmegakaryopoiesis in the form of small size, swollen nuclei, and loose chromatin is also reported [13]. Dyserythropoiesis secondary to megaloblastic changes is mainly seen in patients on ART [14]. The abnormal hematopoiesis could be due to direct HIV infection of bone marrow progenitor cells, the myelotoxic effect of zidovudine, and opportunistic infections like Parvovirus B19 [14]. Interestingly, HIV-1 CXCR4 and CCR5 co-receptor transcripts have been detected in hematopoietic progenitor cells [14]. In our 14 cases with myelodysplasia, 10 were receiving ART therapy and 4 were not on any therapy. Of 10 patients on ART, all had coexistent opportunistic infections (Enterococcus and Molluscum contagiosum: 1, parvovirus: $1, \mathrm{CMV}$ and Candida: 1, M. tuberculosis and CMV: 1, CMV: 1, CMV and Pseudomonas: 1, M. tuberculosis: 1, Candida: 2, and aspergillosis: 1) indicating the multifactorial etiology of HIV myelopathy. Out of 4 patients not receiving ART, 2 had opportunistic infections (Pneumocystis jirovecii and Enterococcus: 1 and atypical mycobacterial infection:1). Erythroid hypoplasia was noted in $4(20 \%)$ cases comprising of parvoviral infection:1, infiltration by Burkitt lymphoma: 1 aspergillosis:1, and markedly hypocellular marrow with CMV infection:1.

HLH in the pediatric age group is usually fatal, and death is inevitable if prompt diagnosis and treatment are not initiated. In the majority of cases, HLH occurs secondary to bacterial infections, tuberculosis, CMV, and Epstein-Barr virus (EBV), though HIV-associated HLH is now being increasingly reported [15]. HLH usually occurs in the late stages of HIV and could be potentiated by opportunistic infections and HIV-associated malignancies [15]. Hemophagocytosis (20\% in our study) could be secondary to HIV and opportunistic infections. Although steroids are the mainstay of treatment in primary and secondary HLH, their role is still controversial in the therapeutic management of HLH in HIV-infected children due to their immunosuppressive effect. Rather, these patients respond well to ART [16]. Of 4 cases, CMV and Candida, PJP and Enterococcus, CMV and Pseudomonas, and CMV alone were present in 1 case each. Though we attempted CMV immunostaining in our cases, we did not find any inclusions.

Myelofibrosis in the pediatric age group is uncommon, and the cases reported are secondary to hematological malignancies, bone marrow failure syndromes, metastatic neoplasms, vitamin D deficiency rickets, auto-immune diseases, and occasionally idiopathic. Myelofibrosis secondary to HIV is extremely rare and could be due to immunological abnormalities associated with HIV [17]. In our study, myelofibrosis was noted in 7 (35\%) cases, of which 1 had multiple bone marrow epithelioid granulomas, 1 had infiltration by Burkitt lymphoma, and 5 cases did not reveal any specific pathology except for megakaryocyte clustering with dysplasia and reactive changes.

Notably, bone marrow could offer a diagnosis of parvoviral infection in the form of giant proerythroblasts with intranuclear inclusions, maturation arrest in the erythropoiesis in a child with anemia, reticulocytopenia, and leukopenia, clinically suspected to be related to ART-induced myelosuppression or lymphoma. The persistence of Parvovirus B19 viremia leading to pure red cell aplasia (PRCA) is uncommon, though an important and treatable cause of anemia in the advanced stage of HIV infection [18].

Involvement of bone marrow by granulomatous inflammation has been frequently reported as a result of low CD4 + T cell count and loss of cell-mediated immunity in HIV/AIDS. Mycobacterial and fungal infections have been reported to induce a granulomatous response in HIV [19]. In our study, 2 (10\%) cases had epithelioid cell granulomas in the marrow, of which 1 had disseminated tuberculosis and was on anti-tubercular therapy (ATT), and the other case had atypical mycobacterial infection diagnosed on peri-pancreatic lymph node fine-needle aspiration cytology. 
In the present study, reactive changes in bone marrow were also identified and included reactive/benign lymphoid aggregates (15\%), an increased number of histiocytes (20\%), and plasma cells (5\%). All these changes are suggestive but not diagnostic of HIV infection. Reactive lymphoid aggregates usually are composed of small mature lymphocytes and a few plasma cells. The presence of small, benign/reactive lymphoid aggregates in the marrow of HIV-infected individuals has been studied, and most of these aggregates are chiefly composed of $\mathrm{T}$ cells or show a mixed $\mathrm{T}$ and $\mathrm{B}$ cell pattern; however, these aggregates are non-specific for HIV and are also found in viral infections including hepatitis $\mathrm{B} / \mathrm{C}$, fungal and mycobacterial infections, autoimmune disorders, and inflammatory disorders [20]. Our study revealed an interstitial increase and aggregates of predominantly $\mathrm{CD} 8+\mathrm{T}$ cells along with a normal to mild interstitial increase in $\mathrm{CD} 20+\mathrm{B}$ cells. $\mathrm{CD} 4+\mathrm{T}$ cells were predominantly reduced in the majority $(78.6 \%)$ of the cases as expected in HIV infection.

There is an overall 60 to 200 -fold increased risk of NHL, and the relative risk of Burkitt lymphoma is 260 -fold higher in HIV as compared to immunocompetent individuals. This increased risk is due to the expansion of polyclonal B cells, as a result of persistent HIV antigen stimulation and/or EBV co-infection. These polyclonal B cells transform to monoclonal $\mathrm{B}$ cells by the acquisition of chromosomal translocations involving chromosome 8 leading to overexpression of the $c-M Y C$ oncogene [21]. In our study, 1 child presented in an advanced stage of Burkitt lymphoma showing diffuse infiltration in bone marrow, developed tumor lysis syndrome, and finally succumbed to his illness. Thus, in the present study, bone marrow examination revealed a wide spectrum of pathological changes including myelodysplasia, variable cellularity, myelofibrosis, hemophagocytosis, granulomatous inflammation, parvoviral infection, infiltration by lymphoma, and reactive lymphoid infiltrates.

\section{Conclusion}

Dyshemopoiesis and non-specific reactive changes were the most common bone marrow changes in our cohort of HIVinfected children, but notably, more actionable entities like granulomatous inflammation, hemophagocytic lymphohistiocytosis, infiltration by lymphoma, and parvovirus infection were also revealed on bone marrow examination. The present study highlights that bone marrow examination provides clinically significant information in pediatric HIV, revealing the underlying cause of hematopoietic abnormalities.

There were a few limitations of the study, the most important being the sample size. Studies including a large number of HIV-infected children undergoing bone marrow examination are required to substantiate the data. Analysis of different cohorts may reveal a different spectrum of hematopoietic abnormalities particularly opportunistic infections and malignancies.

Supplementary Information The online version contains supplementary material available at https://doi.org/10.1007/s12308-021-00471-7.

Author contribution MUS, RS, and SA designed the study. SA, RS, and MUS performed data collection and analysis. The authors (SA, MUS, SS, SN, PS, NK, JA, RD, NV, and AB) performed the morphological assessment. The authors (DS, SS) were involved in clinical management. The first draft of the manuscript was written by SA and MUS. All authors contributed to the writing of the manuscript and critically reviewed and approved the final manuscript.

Data availability Yes.

\section{Declarations}

Ethics approval The study was approved by the Institute Ethics Committee (INT/IEC/2021/SPL-287).

Conflict of interest The authors declare no competing interests.

\section{References}

1. NACO Annual Report; 2018-19. Available from https:// main. mohfw.gov.in/sites/default/files/24\%20Chapter\% 20496AN201819.pdf.

2. Nath A (2017) Pediatric HIV in India: current scenario and the way forward. Indian J Public Health 61:124-130

3. Shah I, Murthy A (2014) Bone marrow abnormalities in HIV infected children, report of three cases and review of the literature. J Res Med Sci 19:181-183

4. Meira DG, Lorand-Metze I, Toro AD, Silva MT, Vilela MM (2005) Bone marrow features in children with HIV infection and peripheral blood cytopenias. J Trop Pediatr 51:114-119

5. Shet A, Mehta S, Rajagopalan N (2009) Anemia and growth failure among HIV- infected children in India: a retrospective analysis. BMC Pediatr 9:37

6. Bhowmik A, Banerjee P (2015) Hematological manifestation in HIV infected children. J Coll Physicians Surg Pak 25:119-123

7. Adetifa IM, Temiye EO, Akinsulie AO (2006) Haematological abnormalities associated with pediatric HIV/AIDS in Lagos. Ann Trop Paediatr 26:121-125

8. Mir N, Costello C, Luckit J, Lindley R (1989) HIV-disease and bone marrow changes: a study of 60 cases. Eur J Haematol 42:339-343

9. Kronenberg A, Seebach JD, Bossart W, Weber R (2001) Polyclonal proliferation of large granular lymphocytes during cytomegalovirus primary infection in a human immunodeficiency virus-infected patient receiving antiretroviral therapy. Clin Infect Dis 33:E34-36

10. McKinney RE Jr, Maha MA, Connor EM, Feinberg J, Scott GB, Wulfsohn M, McIntosh K, Borkowsky W, Modlin JF, Weintrub P et al (1991) A multicenter trial of oral zidovudine in children with advanced human immunodeficiency virus disease. The Protocol 043 Study Group. N Engl J Med 324:1018-1025

11. Bayleyegn B, Woldu B, Yalew A, Asrie F (2021) Magnitude and associated factors of peripheral cytopenia among HIV-infected 
children attending at University of Gondar Specialized Referral Hospital. Northwest Ethiopia. PLoS One 16:e0247878

12. Ramraje SN, Ansari SAH, Kosale S (2016) Diagnostic utility of bone marrow sampling and profile of hematological abnormalities in Indian HIV-infected individuals. Iran J Blood Cancer 8:117-122

13. Delacretaz F, Perey L, Schmidt PM, Chave JP, Costa J (1987) Histopathology of bone marrow in human immunodeficiency virus infection. Virchows Archiv A Pathol Anat 411:543-551

14. Ryu T, Ikeda M, Okazaki Y, Tokuda H, Yoshino N, Honda M, Kimura S, Miura Y (2001) Myelodysplasia associated with acquired immunodeficiency syndrome. Intern Med 40:795-801

15. Tolani D, Shah I (2013) Hemophagocytic lymphohistiocytosis in an infant with probable HIV infection. Indian J Sex Transm Dis AIDS 4:152-153

16. Bansal N, Aggarwal A, Wadhwa N (2016) HIV infection in child presenting as hemophagocytic lymphohistiocytosis. Ann Clin Case Rep 1:1069

17. Lee AC, Fong CM (2012) Autoimmune myelofibrosis as the first manifestation of human immunodeficiency virus infection in an infant. Ann Hematol 91:809-810
18. Koduri PR (2000) Parvovirus B19-related anemia in HIV-infected patients. AIDS Patient Care STDS 14:7-11

19. Zhao X, Sun NC, Witt MD, Keller M, Niihara Y (2004) Changing pattern of AIDS: a bone marrow study. Am J Clin Pathol 121:393-401

20. Naemi K, Brynes RK, Reisian N, Johnston A, Dhillon R, Walavalkar V, Zhao X, Rezk SA (2013) Benign lymphoid aggregates in the bone marrow: distribution patterns of $\mathrm{B}$ and $\mathrm{T}$ lymphocytes. Hum Pathol 44:512-520

21. Johnson DH, Reske TM, Twase H, Ruiz M, Shridhar RJ (2013) HIV-Associated Burkitt Lymphoma: Case Report and Literature Review. Blood 122:5072

Publisher's note Springer Nature remains neutral with regard to jurisdictional claims in published maps and institutional affiliations.

\section{Authors and Affiliations}

\section{Saniya Sharma ${ }^{1}$. Ravinder Kaur Sachdeva ${ }^{2}$ Man Updesh Singh Sachdeva ${ }^{1}$ - Sreejesh Sreedharanunni ${ }^{1}$. Shano Naseem ${ }^{1} \cdot$ Prashant Sharma $^{1}$. Narender Kumar ${ }^{1}$. Jasmina Ahluwalia ${ }^{1} \cdot$ Reena Das $^{1} \cdot$ Neelam Varma $^{1}$. Amanjit Bal ${ }^{3} \cdot$ Deepti Suri ${ }^{2} \cdot$ Surjit Singh ${ }^{2}$}

\author{
Saniya Sharma \\ drsaniya.sharma@gmail.com \\ Ravinder Kaur Sachdeva \\ drravinderpgi@yahoo.co.in \\ Sreejesh Sreedharanunni \\ dr.s.sreejesh@gmail.com \\ Shano Naseem \\ shanonaseem@yahoo.co.in \\ Prashant Sharma \\ prashant.sh@gmail.com \\ Narender Kumar \\ narenderkalson@gmail.com \\ Jasmina Ahluwalia \\ jasminapgi@gmail.com \\ Reena Das \\ reenadaspgi@hotmail.com
}

Neelam Varma

varmaneelam@yahoo.com

Amanjit Bal

docaman5@hotmail.com

Deepti Suri

suri.deepti@pgimer.edu.in

Surjit Singh

surjitsinghpgi@ rediffmail.com

1 Department of Hematology, Postgraduate Institute of Medical Education and Research, Chandigarh 160012, India

2 Department of Pediatrics, Postgraduate Institute of Medical Education and Research, Chandigarh, India

3 Department of Histopathology, Postgraduate Institute of Medical Education and Research, Chandigarh, India 